\title{
Collective Efficiency in Two-Sided Matching
}

\author{
Tomoko Fuku, Akira Namatame ${ }^{1}$ and Taisei Kaizouji ${ }^{2}$ \\ ${ }^{1}$ \{g43036,nama\}@nda.ac.jp \\ Department of Computer Science, National Defense Academy, Yokosuka, Kana- \\ gawa, 239-8686, Japan \\ ${ }^{2}$ kaizoji@icu.ac.jp Division of Social Sciences, International Christian University, \\ 3-10-2 Osawa, Mitaka, Tokyo, 181-8585 Japan
}

\begin{abstract}
:
Gale and Shapley originally proposed the two-sided matching algorithm, Deferred Acceptance Algorithm (DA). This is very brilliant method, but it has a demerit which produces. That is if men propose, it produces stable matching which is the best for men and the worst for women, and vise versa.

In this paper, we propose a new algorithm with compromise that produce the balanced matching which are almost optimal for both sides. It is an important issue how far agents seek their own interests in a competitive environment. There are overwhelming evidences that support peoples are also motivated by concerns for fairness and reciprocity. We will show that compromise which is individually irrational improves the welfare of the whole groups. The reasonable compromise level is obtained as the function of the size of the group so that the social utility should be maximized. We also obtain large-scale properties of the proposed algorithm.
\end{abstract}

\section{Introduction}

Some researchers have started to take a direct role in issues of designing market, e.g. labor market, a venue for bilateral trading require a proper matching. But, in considering the design of markets, is extremely complex. Markets evolve, but they are also designed. The complexity of market design comes from many factors, especially strategic behaviors of participants. A market is two-sided if there are two sets of agents, and if an agent from one side of the market can be matched only with an agent from the other side. One of the main functions of many markets is to match one kind of agent with another: e.g. students and colleges, workers and firms, marriageable men and women. A two-sided matching model is introduced by Gale and Shapley(1962), and they invented the deferred acceptance algorithm.

One of the basic problems in societies is to match one kind of agent with another, e.g. marriageable men and women students and colleges, workers and firms. A two-sided matching model was introduced by Gale and Shapley, and they focused on college admissions and marriage. They proposed that a matching (of students and colleges, or men and women) could be regarded as stable only if it left no 
pair of agents on opposite sides of the market who were not matched to each other but would both prefer to be.

A natural application of two-sided matching models is to labor markets. Shapley and Shubik (1972) showed that the properties of stable matching are robust to generalizations of the model which allow both matching and wage determination to be considered together. Kelso and Crawford (1982) showed how far these results can be generalized when firms, for example, may have complex preferences over the composition of their workforce. Two-sided matching models have proved useful in the empirical study of labor markets, starting with the demonstration in Roth (1984). Subsequent work has identified natural experiments which show that labor markets organized so as to produce unstable matching suffer from certain kinds of difficulties which are largely avoided in comparable markets organized to produce stable matching. This work combines the traditions of cooperative and noncooperative game theory, by considering how the strategic environment faced by market participants influences the stability of the resulting market outcome.

Much of two-sided matching theory is concerned with determining the conditions under which stable matching exist, and with what algorithms these matching can be achieved. A two-sided matching could be regarded as stable if it left no pair of agents on opposite sides of the market was not matched to each other but would both prefer to be. The relationship between the concept of Pareto optimality and the stability of a matching has been also investigated. Pareto optimality requires that no change exists that betters every individual in the population. The concept of a stable matching is stronger than that of a Pareto optimal matching, in that every stable matching is Pareto optimal, but not every Pareto optimal matching is stable. Pareto optimality requires that no two individuals wish to elope together and would receive the consent of their partners. Stable matching, by contrast, requires that no two individuals wish to elope together, whether or not their partners would consent.

For instance let consider two groups of marriageable men and women. A stable matching $\mu$ is defined as $M$-optimal if no male prefers any other stable matching. F-optimality is defined analogously. However, the M-optimal matching is not only the best stable matching for the males, it is also always the worst stable matching for the females. In fact, male and female preferences conflict in this way over any pair of stable matching, not just the $M$ - and F-optimal ones.

In this paper, we propose a new algorithm for two-sided matching with some compromise. We discuss the self-interested hypothesis vs. human sociality hypothesis. It is an important issue such as how far agents seek their own interest in a competitive environment? There are overwhelming evidences that support peoples are also motivated by concerns for fairness and reciprocity. We showed that compromise, an individually irrational behavior, improves the welfare of others. We also obtain large-scale properties of some two-sided matching algorithms. We show some compromises of individuals increase global welfare. The optimal compromise level is designed so that the social utility is maximized. 


\section{A Formulation of Two-sided Matching Problem}

There are two disjoint sets of agents, groups of men $=\left\{m_{1}, \ldots, m_{n}\right\}$, and women $=$ $\left\{f_{1}, \ldots, f_{n}\right\}$. Associated with each side is the number of positions they have to offer. Agents on each side of the market have (transitive and strict) preferences over agents on the other side, with presented a simple model in which college applicants have ordinal preferences over schools, and colleges have ordinal preferences over applicants. How, given these preferences, could college applicants be matched to schools so as to leave both the students and the colleges as satisfied as possible? The authors derived a clever algorithm (described below) designed to create efficient pairings. Their algorithm matches students and schools in such a way that no student wishes to leave her current school for an alternative institution that would be willing to admit her. Subsequent authors expanded upon Gale and Shapley's work, extending their theoretical framework while applying two-sided matching theory to problems ranging from labor markets to human courtship and marriage.

Consider a population, each member of which falls into one of two sets: the set of all males $M=\left\{m_{1}, m_{2}, m_{3}, \ldots, m_{n}\right\}$ and the set of all females $F=\left\{f_{1}, f_{2}, f_{3}, \ldots, f_{n}\right\}$. Let each individual $m_{i}$ or $f_{j}$ have a list of strict pairing preferences $P$ over the individuals in the other set. For example, a female $f_{j}$ might have preferences $P\left(f_{j}\right)=\left\{\mathrm{m}_{1}\right.$, $\mathrm{m}_{4}, f_{j}, \mathrm{~m}_{3}, \mathrm{~m}_{2\}}$, meaning that male $\mathrm{m}_{1}$ would be her best choice, $\mathrm{m}_{4}$ would be her second choice, and she would rather remain 'single' (represented by pairing with herself, $f_{j}$ ) than form a pair with either $\mathrm{m}_{2}$ or $\mathrm{m}_{3}$.

A matching is simply a list of all the pairings in the population (where having oneself for a mate means that one remains single). We indicate the mate of an individual $\chi$ under matching $\mu$ by using $\mu(\chi)$ for short. Now we are ready to consider the notion of the stability of a matching(Knuth(1962)). An individual is said to block the matching $\mu$ if he or she prefers remaining single to taking the mate assigned by $\mu$. A pair $m$ and $f$ are said to block the matching $\mu$ if they are not matched by $\mu$, but prefer one another to their mates as assigned by matching $\mu$. Put another way, given matching $\mu$, a blocking pair is a pair that would willingly abandon their mates as determined by $\mu$ and elope instead with one another. Finally, the matching $\mu$ is defined as stable if it is not blocked by any pair of agents.

\section{Deferred Acceptance Algorithm and Its Properties}

What happens when preferences are not uniform? One of the most remarkable results from two-sided matching theory is that, even under non-uniform preferences, a stable matching (or set of stable matching) exists in every monogamous matching system. To prove this, it is sufficient to describe an algorithm by which a stable matching can be constructed for any such system. We need not suppose that pairing actually occurs by this algorithm in the system we are considering. Rather, the algorithm simply serves as a tool in the proof that a stable matching exists. Below, we 
outline the deferred acceptance algorithm (DA).The matching procedure then proceeds repeatedly through the following steps.

$<$ Step 1> Each male not currently engaged displays to his favorite female that has not already rejected him. If no acceptable females remain, he remains unmated.

$<$ Step 2> Each female who has received one or more courtship displays in this round rejects all but her highest-ranked acceptable male. This may involve rejecting a previously engaged male.

$<$ Step $\mathrm{n}>$ After a large number of rounds, no new displays will be made.

At this point, the algorithm terminates. All females are paired with the male to whom they are currently engaged; individuals not engaged remain unmated. The matching $\mu$ generated in this way is easily seen to be stable. No male wishes to leave his mate at $\mu$ for a female who prefers him to her mate at $\mu$, because each male reached his current mate by sequentially courting females in order of preference. No female wishes to leave her mate at $\mu$ for a male who prefers her to his mate at $\mu$, because she will have already received a courtship display from any male who is not matched to a female that he prefers to her.

Reversing the algorithm, so that the females display and the males accept or reject court-ships, will also lead to a stable matching; this matching may be a different one than that found by the male-courtship form of the algorithm. However, the set of individuals remaining unmated is the same in every stable matching of any given monogamous mating system.

As above-mentioned, Deferred Acceptance Algorithm is defined (1) Manoptimal stable matching or (2) Woman-optimal stable matching. That is (1) the matching $h_{M}$ produced by the deferred acceptance algorithm with men proposing is the M-optimal stable matching, or, (2) the W-optimal stable matching is the matching $h_{w}$ produced when the women propose (Gale and Shapley). And it's emerged that the best outcome for one side of the market is the worst for the other. i.e. Moptimal stable matching is the worst for women. And W-optimal stable matching is the worst for men (Knuth (1972)). It may be helpful to look at this problem with some concrete example. Consider a group of women(Ann, Betty and Carol) and a group of men (Dave, Eddy and Frank). The preferences of those are given in Table 1 (3 represents the highest preference and 1 represents the lowest).

In this matching system, there are two stable matching. One (call it $\mu_{1}$ ) pairs Betty with Dave, Ann with Eddy, and Carol with Frank. The other, $\mu_{2}$, pairs Betty with Dave, Ann with Frank, and Carol with Eddy. Any other matching will allow at least one blocking individual or pair. In fact, since Betty and Dave are one another's best choices, any matching $\mu_{-}$which does not pair them together will be blocked by this pair. 
Table 1. Preferences relation

\begin{tabular}{|c|c|c|c|}
\hline & Dave & Eddy & Frank \\
\hline \multirow{2}{*}{ Ann } & 2 & 2 & 2 \\
\hline & 3 & 1 & 2 \\
\hline \multirow{2}{*}{ Betty } & 3 & 3 & 1 \\
\hline & & 2 & 1 \\
\hline \multirow{2}{*}{ Carol } & 1 & 1 & \\
\hline & 1 & 3 & 2 \\
\hline
\end{tabular}

Table 2. Preference relation Betty and Dave are removed from Table.1

\begin{tabular}{|c|c|c|}
\hline & Eddy & Frank \\
\hline \multirow{2}{*}{ Ann } & 2 & 1 \\
\hline & 1 & 2 \\
\hline \multirow{2}{*}{ Carol } & 1 & 2 \\
\hline & 2 & 1 \\
\hline
\end{tabular}

If we are interested in yielding all stable matching, we can remove Betty and Dave from the preference list, yielding the reduced preference list given in Table 2.

When all preferences are uniform - that is, when all males have the same preferences over females and vice versa - it is easy to see that a unique stable matching exists. To see this for monogamous matching systems, label the members of each sex by the preferences of the other sex (so that the best-ranking male $m_{1}$ is the best choice of the females, $\mathrm{m}_{2}$ is the second choice, etc.). Under this system, the only possible stable matching will be approved.

\section{Proposed of New Algorithms}

Before we state our algorithms, we define utility measures. In this section, we propose two new algorithms based on the hypothesis bounded rationality. In this paper, we propose a new algorithm for two-sided matching some compromise at individual levels. We also obtain large-scale properties of the proposed algorithm. It is an important issue how far agents seek their own interests in a competitive environment. There are overwhelming evidences that support peoples are also motivated by concerns for fairness and reciprocity. We will show that compromise which is individually irrational improves the welfare of the whole groups. The reasonable com- 
promise level is obtained as the function of the size of the group so that the social utility should be maximized.

\section{$<$ Definition>}

There are two disjoint sets of agents, groups of men $=\left\{m_{1}, \ldots, m_{n}\right\}$, and women $=$ $\left\{f_{1}, \ldots, f_{n}\right\}$. Associated with each side is the number of positions they have to offer. Agents on each side of the market have (transitive and strict) preferences over agents on the other side, with presented a simple model in which college applicants have ordinal preferences over schools, and colleges have ordinal preferences over applicants.

$\mathrm{N}$ : the size of each group

$\mathrm{T}$ : the preference level of the partner to be matched $(1 \leq T \leq N)$

(1) Individual utility(utility of agent $i$ )

(2) Utility of a pair of agent $i$ and agent $j$

$$
U_{i}=1-T_{i} / N
$$

$$
P_{i j}=U_{i} \times U_{j}
$$

(3) Social utility

$$
S=\sum P_{i j}
$$

We propose a new algorithm based on concept of compromise of individual.

\subsection{Matching with Aggregated Preference : MAP}

When each group is composed of $\mathrm{N}$ person, stable matching exists according to $\mathrm{N}$. In addition, the more the scale of the group increases, the more the number of stable matching increases explosively. Because a stable matching exists a lot, according to the match dissatisfaction will be caused in a lot of people. To solve this problem, it thought the only match was produced. It's < Matching with Aggregated Preference : MAP>.

The preference order is decided in the entire group. The matching is produced based on it. That is, the negotiation among individuals is not admitted, it is a topdown match method. The sum total of the score of women's preference order for each men $m_{i} \in M$ is obtained. To similar, the sum total of the score of men's preference order for each women $w_{j} \in W$ is obtained. The pair of order is made from the person with a high total score.

\subsection{Modified Deferred Algorithm with Compromise : MDA}

Another algorithm is $<$ Modified Deferred Algorithm with Compromise>. It's natural that the optimal strategy is to behave as if the individuals can profit the maximum. But like prisoner's dilemma game everyone knows, the social best optimum is to act in concert. Similarly, in the marriage problem we wonder if the social 
optimal point exists when the individuals compromise acceptable level. It happens that no compromise result in the lowest social utility because of the large difference satisfaction with disappointment, and increasing matching paired slowly. Moreover, it is sure that more compromise result the lowest social utility because many individuals can not be satisfied the outcome. However we can compromise at individual level properly, most of individuals somewhat can increase their satisfaction. Necessarily, we suppose that we can get the most social utility by increasing the pairs with somewhat satisfaction. For the present, it delays the discussion of this algorithm's efficacy till later, we thought about the value of the most optimal compromising level. It follows the simulation result.

$<$ Step1 > Each man proposes to his $1^{\text {st }}$ choice. Proposed woman accepts the proposal if it is within the acceptable level, otherwise she rejects it (holding is not permitted).

$<$ Step $\mathrm{k}>$ Each woman who is not matched increases the acceptable level by one. Any man who was rejected at step k-1 makes a new proposal to his most preferred woman mate who has not yet rejected him. A proposed woman accepts the proposal within the acceptable level, otherwise she rejects it.

We lock the value of $\mathrm{N}(=1000)$, agents number. We increase the compromise level (x-axis) by 0.1 , and plot the calculated value. We can get the parabola like Figure 1. Therefore, maximum point of social utility becomes the most optimum compromise level point.

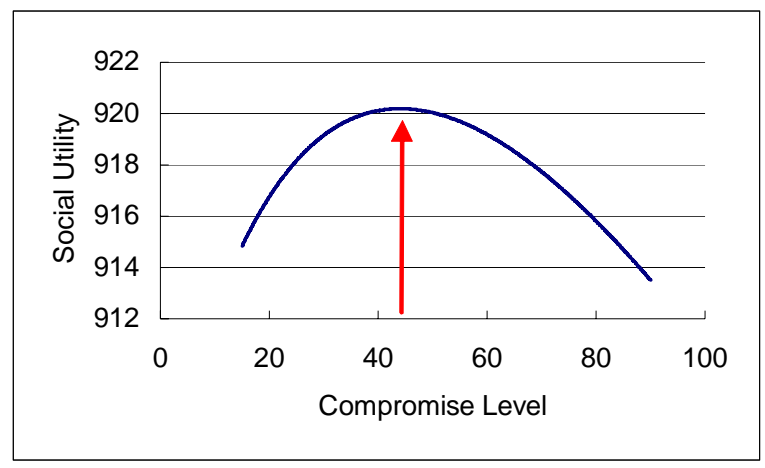

Figure 1. The relationship between compromise level and Social Utility ( $\mathrm{N}=1000)$ 


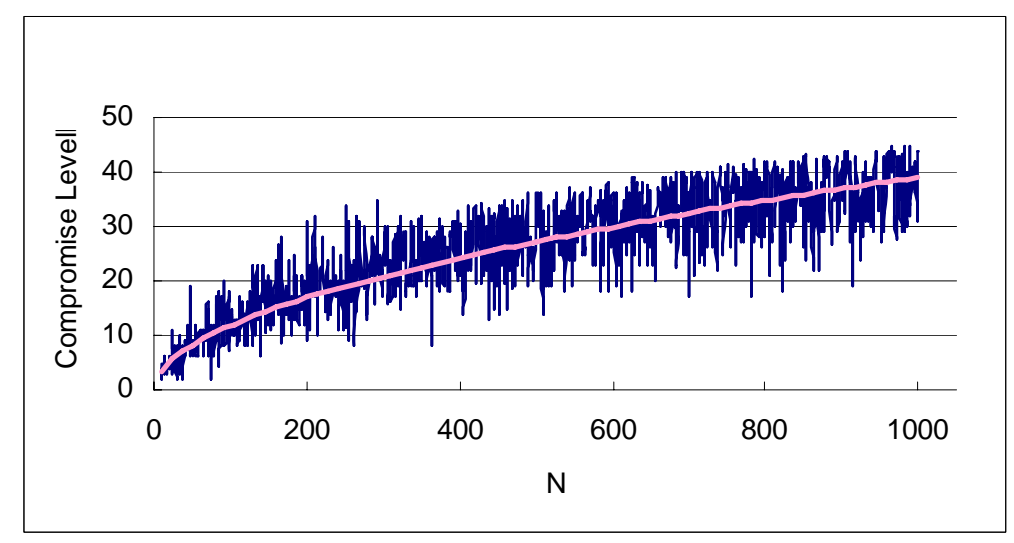

Figure 2. The relationship between the compromising level and $N_{1}$ the number of agent of each group

The relation between compromise (C) and $\mathrm{N}$ is approximation by

$$
C=1.077 N^{0.5197} \cong \alpha \sqrt{N} \quad(\alpha \cong 1.2)
$$

\section{Simulation Results}

We generate the preference list of the agents at random. An agent belong to the each group is matched using three algorithm simulation (DA, MAP, MDA). Then, we compare about the distribution of availability about each agents or blocking pair, and the social utility. It follows the simulation result $(\mathrm{N}=1,000)$, that is the distribution of availability.

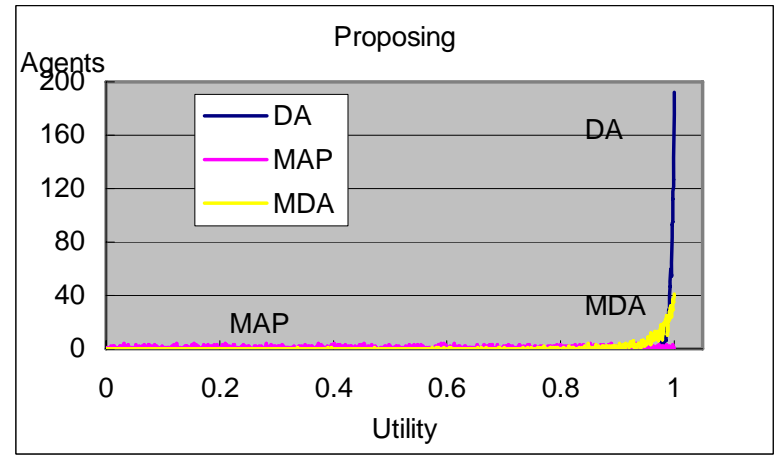

Figure 3. The utility distribution of men who propose to women

These figures describe the utility distribution of each side. MAP is how to decide of which everyone consents because the preference list of the individual is consoli- 
dated and it decides the matching. However, the person with low effect exists from the person with high effect almost thoroughly, and, as a result, it becomes the one with considerably low effect of the entire society. Therefore, it is understood that a lot of top-down methods of not admitting the individual to negotiate invent a dissatisfied pair.

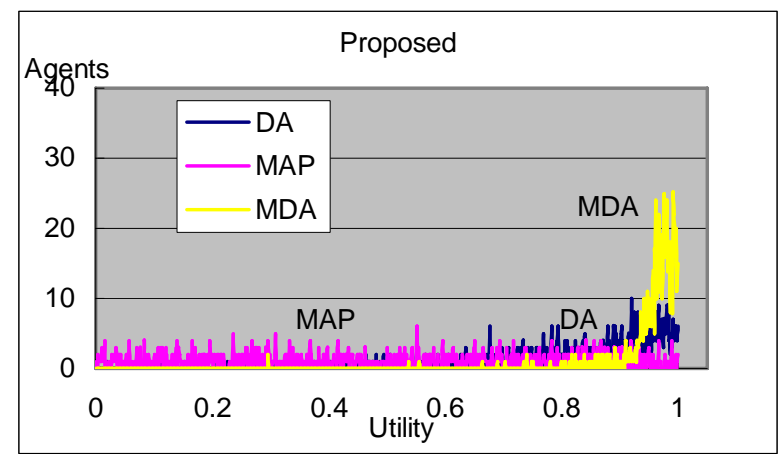

Figure 4. The utility distribution of women who are proposed from men

To be sure, the Figure 3 shows that there are many agents near 1 utility in DA. Opposite, the Figure 4 show that agents utility distributes flatly. Moreover, the yaxis maximum value of the Figure 4 is 40, in contrast Figure 3 is 200. These are why DA is called the M-optimal (Proposing side optimal) algorithm.

Then, Figure 4 shows that MDA distributes more flatly than DA. But the utility of both of side are modestly similar. In addition, almost of agents gather around 1 . So, they can assume Pair-optimal and high utility. Additionally, the utility of Pair follows Figure 5.

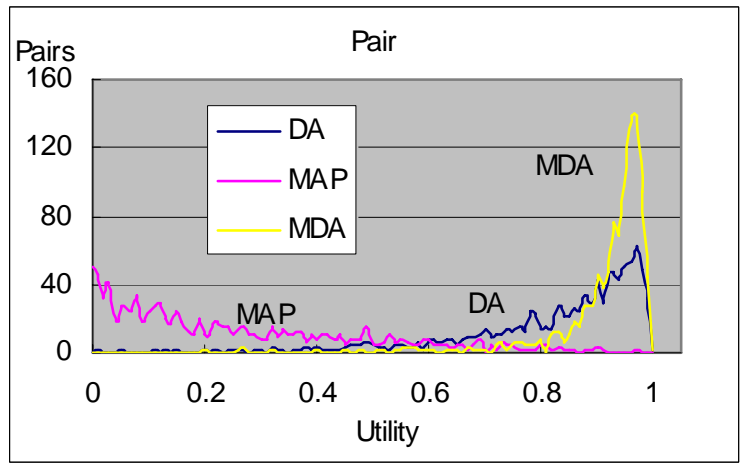

Figure 5. Utility distribution of the Pairs 
This figure shows the distribution of utility which pairs have. Almost pairs satisfy the outcome of the matching. Especially, MDA heaps many pairs around utility 1. As pair utility, DA is not as well as proposing utility. That is proposed agents must accept all proposing without reference to the self-preference lists. It is natural that many proposing agents can satisfy the outcome, but proposed agents can not. In other word, DA have many satisfied proposing agents, few proposed agents. Consequently, the utility of the Pair hold lower than MDA. Then, we can get the higher utility of pair with some compromises or borderlines.

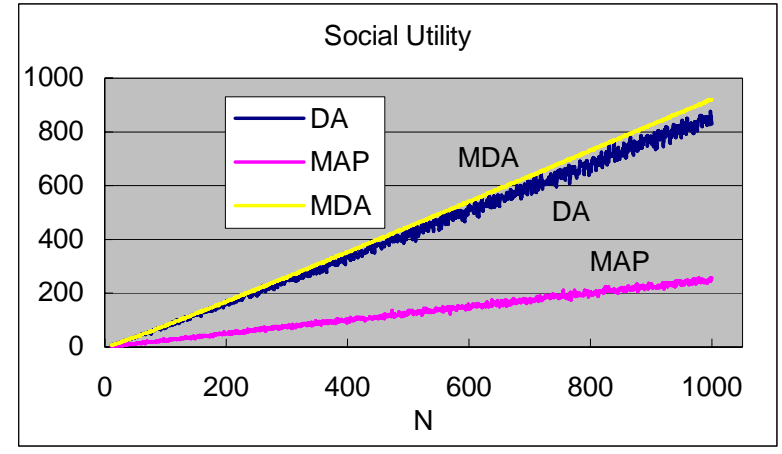

Figure 6. Comparison of social utility of three algorithms

We can describe the social utility by liner function. Figure 6 shows social utility in MDA is higher than in DA. Especially, the more the circle scale is bigger. When the circle scale is small, in MDA social utility can be lower, because compromise level is large percentage against $\mathrm{N}$. In small circle scale social utility in DA can be higher than MDA. But we can get high social utility in MDA in large circle.

\section{Theoretical Consideration of the Optimal Compromise Level}

In section4, we obtained the relationship between the optimal compromise level and the population size by simulation which is obtain in (3.2). In this section, we obtain it theoretical analysis. We define the following terminologies:

$$
\begin{aligned}
& g(t) \text { : The number of paired couples at the t step } \\
& f(t) \text { : The number of unmatched agents at the t step } \\
& C \text { : The compromise level }
\end{aligned}
$$

We have the following initial conditions:

$$
g_{(0)}=C, f_{(0)}=N-C
$$

From the definition, we have the following recursive equations.

$$
g(t)=f(t-1) \times(C+t) / N \times f(t-1) / N
$$

Fore the number of unmatched pair at the t-th step is obtained as 


$$
f(t)=f(t-1) \times\left(1-f(t-1) \times(C+t) / N^{2}\right.
$$

\section{$<$ The proof $>$}

In the case, the contents of preference lists each agents have are at random, paired couple arise at random, too. Each side scale is $N$ agents, the compromise level is $C$, we suppose. $g$ is the number of paired couples on the step, $f$ describes the number of other free agents.

$<$ Step1> Each $N$ agents on one side propose the other side best partner, then, the probability of accepting proposal is $C / N$. So, on the first step, the number of paired-couple is

$$
g_{(0)}=N \times C / N=C,
$$

and the number of free agents (who are still single, non-couple) is

$$
f_{(0)}=N-g_{(0)}=N-C \text {. }
$$

$<$ Step $\mathrm{t}>$ The agents of $f(t-1)$ propose the $t^{\text {th }}$ partners of each preference lists. The probability that the $t^{\text {th }}$ partners of each preference lists is still free is

$$
f(t-1) / N \text {, }
$$

and the probability of the accepting proposal is

$$
(C+t) / N \text {. }
$$

Then, the number of paired couple on the step $t$ is

$$
g(t)=f(t-1) *(C+t) / N^{*} f(t-1) / N^{2} .
$$

On this step, the number of free agents is

$$
f(t)=f(t-1)-g(t) .
$$

That is

$$
f(t)=f(t-1) *\left(1-f(t-1) *(C+t) / N^{2}\right.
$$

If we solve the recursive equation (6.3), we have

$$
C \cong 1.3 \sqrt{N} \text {. }
$$

Therefore we could get the nice approximation in (4.2).

\section{Conclusion}

Deferred Acceptance has universally used since it suggested for 40 years. To be sure, this is very brilliant method for two sided matching, but it has a demerit which is a M-optimal algorithm. This research pursued that we produced the moderate equality for each side. We suggested two algorithms. We use agent-based simulation, we inspect each algorithm's quality and social utility. We could show pairoptimal matching by using MDA. I mean, to be sure compromise behavior is an individually irrational, but is not in macro world. Compromise (Patient) behavior link collaboration, and eventuate high efficiency. The main reason is sure that much compromise profit better partner not the best partner. For many agents, the best 
partner exists only one, but better partner exist more. Many agents can moderately satisfy, therefore, social utility is getting better globally.

In addition, the balance of proposing side and proposed side can keep well. It is sure that proposing side utility gets down totally, but commensurately proposed side utility is getting better. Globally, we can get higher social utility outcome. It may natural that compromise or borderline profit social utility globally, nevertheless DA is the best individually. But we will not insist the new algorithms performance.

We suppose, when we design the rule or institution in many of two sided matching problems like to students and colleges, workers and firms, marriageable men and women, compromise which is irrational behavior can bring higher social payoff for many people and the rule itself.

In conclusion, self-interested hypothesis vs human sociality hypothesis folows. How far agents seek their own interest in a competitive environment? There are overwhelming evidences that support people are also motivated by concerns for fairness and reciprocity. For this mentioned above question, we showed that compromise, an individually irrational behavior, improves the welfare of others.

\section{References}

[1] Crawford, V.P. and Knoer, E.M. 1981. Job matching with heterogeneous firms and workers. Econometrica, 49: 437-450.

[2] Cunningham, E.J.A. and Birkhead, T.R. 1988. Sex roles and sexual selection. Anim. Behav., 56: 1311-1321.

[3] Gale, D. and Shapley, L.S. 1962. College admissions and the stability of marriage. Am. Math.Monthly, 69: 9-15.

[4] Gerd Gigerenzer, and Peter M. Todd. 1999. SIMPLE HEURISTICS THAT MAKE US SMART.

[5] Knuth, D.E. 1976. Stable Marriage and its Relation to other Combinatorical Problems. CRM Proceedings and Lecture Notes Vol. 10, English language edition. Providence, RI: American Mathematical Society. Originally published in French under the title Mariages stables et leurs relations avec d'autres problèms combinatoires.

[6] Marie-jose Omero, Michael Dzierzawa, Matteo Marsili, Yi-Cheng Zhang 2004. Scaling Behavior in the Stable Marriage Problem. cond-mat/9708181

[7] Michael Dzierzawa, Marie-jose Omero, 2000. Statistics of stable marriage, cond-mat 19708181

[8] Mongell, S. and Roth, A.E. 1991. Sorority rush as a two-sided matching mechanism. Am. Econ. Rev.,81: 441-464.

[9] Roth, A.E. and Sotomayor, 1990. Two-sided Matching: A Study in Game-theoretic Modeling and Analysis. Econometric Society Monographs No. 18. New York: Cambridge University Press.

[10] Shapley, L.S. and Shubik, M. 1972. The assignment game I: The core. Int. J. Game Theory, 1:111-130. 